\title{
QTL Mapping for Gray Leaf Spot Resistance in a Tropical Maize Population
}

L. Liu, Y. D. Zhang, H. Y. Li, Y. Q. Bi, L. J. Yu, and X. M. Fan, Institute of Food Crops, Yunnan Academy of Agricultural Sciences/Yunnan TianRui Seed Company, Ltd., Kunming 650200, Yunnan Province, China; J. Tan, School of Agriculture, Yunnan University, Kunming 650091, Yunnan Province, China; D. P. Jeffers, CIMMYT Yunnan Office/Institute of Food Crops, Yunnan Academy of Agricultural Sciences, Kunming 650200, Yunnan Province, China; and M. S. Kang, Department of Plant Pathology, Kansas State University, Manhattan KS 66506-5502

\begin{abstract}
Liu, L., Tan, J., Zhang, Y. D., Li, H. Y., Bi, Y. Q., Yu, L. J., Jeffers, D. P., Kang, M. S., and Fan, X. M. 2016. QTL mapping for gray leaf spot resistance in a tropical maize population. Plant Dis. 100:304-312.

A tropical gray leaf spot (GLS)-resistant line, YML 32, was crossed to a temperate GLS-susceptible line, Ye 478 , to produce an $\mathrm{F}_{2: 3}$ population for the identification of quantitative trait loci (QTL) associated with resistance to GLS. The population was evaluated for GLS disease resistance and flowering time at two locations in Yunnan province. Seven QTL using GLS disease scores and six QTL using flowering time were identified on chromosomes $2,3,4,5$, and 8 in the YML $32 \times$ Ye 478 maize population. All QTL, except one identified on chromosome 2 using flowering time, were overlapped with the QTL for GLS disease scores. The

results indicated that QTL for flowering time in this population strongly corresponded to QTL for GLS resistance. Among the QTL, qRgls.yaas8-1/qFt.yaas-8 with the largest genetic effect accounted for 17.9 to 18.1 and 11.0 to $21.42 \%$ of variations for GLS disease scores and flowering time, respectively, and these should be very useful for improving resistance to GLS, especially in subtropical maize breeding programs. The QTL effects for resistance to GLS were predominantly additive in nature, with a dominance effect having been found for two QTL on the basis of joint segregation genetic analysis and QTL analysis.
\end{abstract}

Gray leaf spot (GLS), caused by Cercospora zeae-maydis (Tehon and Daniels 1925) and C. zeina-maydis (Crous et al. 2006; Liu and $\mathrm{Xu} 2013$ ), is a global threat to maize production (Katwal et al. 2013; Ward et al. 1999). In the 1960s and 1970s, the disease became of concern in the eastern United States. In the 1980s and 1990s, as reduced tillage gained popularity, GLS became a noticeable problem in most of the maize-growing areas of the midwestern and eastern United States. Now, the disease has become an international problem, from Africa to Asia and in North and South America, and has caused huge maize yield losses (Crous and Braun 2003). Documented yield losses of maize attributed to GLS varied from 11 to $69 \%$ (Ward et al. 1999), with estimated losses as high as $100 \%$ when severe epidemics occurred. The GLS damage has been linked to loss of photosynthetic capability and premature plant death (Latterell and Rossi 1983; McGee 1988). Because of broad distribution of the disease and severity of losses to maize production, GLS control has become a major breeding objective in many maize improvement programs worldwide (Menkir and Ayodele 2005; Nzuve et al. 2013).

The most common GLS control methods include the application of fungicides, cultural practices (i.e., crop rotation and field sanitation), and host resistance (Ward et al. 1999). Because all other methods are not highly effective or are detrimental to the environment, host resistance, which is considered to be environmentally friendly, inexpensive, and highly effective, seems to be the best means of reducing yield losses caused by GLS (Shi et al. 2014).

Introgression of resistance genes from donors into elite maize germplasm has proved to be effective in improving disease resistance (Gordon et al. 2004). Previous studies have shown additive genetic effects to be more important than dominance effects (Clements

Corresponding author: X. M. Fan; E-mail: xingmingfan@163.com

L. Liu and J. Tan contributed equally to this work.

*The $\boldsymbol{e}$-Xtra logo stands for "electronic extra" and indicates that one supplementary table is published online.

Accepted for publication 4 August 2015.

http://dx.doi.org/10.1094/PDIS-08-14-0825-RE

(c) 2016 The American Phytopathological Society et al. 2000; Coates and White 1994; Donahue et al. 1991; Elwinger et al. 1990; Gevers et al. 1994; Gordon et al. 2006). Other studies have indicated that resistance to GLS in maize germplasm was controlled by multiple genes (Balestre et al. 2012; Danson et al. 2008; Donahue et al. 1991; Gevers et al. 1994; Huff et al. 1988). Thus, it is quite logical to search for molecular markers linked to quantitative trait loci (QTL) offering resistance to GLS to speed up the development of resistant inbreds or hybrids through marker-assisted selection (MAS). Knowledge of genetic effects and nature of resistance QTL is important for introducing favorable alleles into susceptible materials and for optimal utilization of genetic variability (Pozar et al. 2009).

Several important QTL for GLS resistance have successfully been identified in maize under various environments in different resistant germplasms (Balint-Kurti et al. 2008; Danson et al. 2008; Saghai Maroof et al. 1996; Zhang et al. 2012). For example, Saghai Maroof et al. (1996) identified QTL for resistance to GLS on chromosomes 1, 4 , and 8 in the Va14 $\times$ B73 cross and these QTL explained 35.0 to $56.0,14.3$, and 7.7 to $11.0 \%$, respectively, of the variation for resistance to GLS. Gordon et al. (2004) identified two QTL associated with GLS resistance on chromosomes 2 and 4, which together explained 40 to $47 \%$ of the total GLS resistance variation in an $F_{2: 4}$ population derived from the VO613Y $\times$ Pa405 cross. Similar reports have been published by other researchers, with resistance QTL also identified on chromosomes 1, 3, 5, 7, 9, and 10 (BalintKurti et al. 2008; Benson et al. 2015; Berger et al. 2014; Clements et al. 2000; Lehmensiek et al. 2001; Messmer et al. 2009; Pozar et al. 2009; Xu et al. 2014; Zhang et al. 2012; Zwonitzer et al. 2010). Because many GLS resistance QTL have been detected in different populations and environments, Shi et al. (2007) constructed an integrated GLS resistance QTL map and found that 26 real and 7 consensus QTL were located in 1.06, 2.06, 3.04, 4.06, 4.08, 5.03, and 8.06 bins.

Previous studies reported that GLS resistance correlated to flowering time and later-maturing plants showed high resistance (BalintKurti et al. 2008; Benson et al. 2015; Bubeck et al. 1993; Clements et al. 2000; Zwonitzer et al. 2010). The flowering times were highly associated with GLS resistance in the KB population developed from the tropical inbred Ki14 compared with IBM population developed from the temperate inbred B73 (Zwonitzer et al. 2010). Messmer et al. (2009) found that QTL corresponding to flowering time in 
the population of CML444 $\times$ SC Malawi did not overlap with GLS resistance QTL identified in that study, although it was close to the main-effect GLS resistance QTL observed in multiple environments. Further studies demonstrated that QTL for flowering time in the population of CML444 $\times$ SC Malawi did not correspond to GLS resistance QTL (Berger et al. 2014).

Although many putative GLS resistance QTL have been identified, subtropical and tropical maize possess high levels of unexplored genetic diversity, and it is valuable to identify additional sources of resistance in elite breeding germplasm utilized in these environments. Because of their ability to combine differences among different maize lines, and their capacity to develop elite hybrids, different GLS-resistant germplasms and QTL for GLS resistance existing in those resources need to be explored for their proper utilization in hybrid maize breeding programs (Chevin and Hospital 2008). With the identification of good combiners between Suwan 1 (tropical germplasms) and Reid or non-Reid (temperate germplasm) heterotic groups (Fan et al. 2014), tropical GLS-resistant germplasm can be very valuable in hybrid development, especially when this can be utilized in subtropical environments to increase yields in the presence of many biotic and abiotic stresses. It's important to screen additional germplasm, especially tropical maize germplasm, for GLS resistance and identify their resistance QTL.

A tropical inbred line, YML 32, which is highly resistant to GLS, was developed at the Institute of Food Crops, Yunnan Academy of Agricultural Sciences, from Suwan 1. Suwan 1 not only propelled Thailand to become the fourth largest maize exporting country in 1986 but it has also been used as a source material or as direct release in Africa, Asia, Oceania, and South America (Sriwatanapongse et al. 1993). In southwest China, including Yunnan, Guangxi, and Guizhou, YML 32 has been successfully used as a source material for the development of GLS-resistant hybrids such as Yunrui 1. Temperate inbred line Ye 478, which is highly susceptible to GLS, is one of the most widely used parental lines in hybrid maize breeding programs in China. To date, more than 40 maize hybrids released for production in China have been developed from Ye 478. Among the released hybrids, Zhengdan 958, formed from a derived parental line, is one of the most influential hybrids and covered 38.76 million ha in China in 2006 (Dai and E 2010). We have found that YML 32 always produces GLS-resistant hybrids, and the genetic analysis of GLS resistance and GLS-resistant QTL identification would provide useful information for development of maize hybrids and the development of a MAS breeding program. In this study, the tropical inbred line YML 32 (resistant to GLS) was crossed to the susceptible temperate inbred line $\mathrm{Ye} 478$ to produce an $\mathrm{F}_{2: 3}$ population for the identification of QTL associated with resistance to GLS. The objectives were to (i) study the genetic effects of QTL imparting resistance to GLS, (ii) identify QTL associated with resistance to GLS in tropical maize germplasm; and (iii) verify whether the flowering time QTL in this tropical population were associated with the GLS resistance.

\section{Materials and Methods}

Plant materials. In 2009, the resistant inbred YML 32, used as female parent, was crossed with the highly susceptible inbred Ye 478 at Jinghong, Yunnan, China in the winter season. In 2010, The $F_{1}$ was selfed in Kunming, Yunnan, during the summer season. The $F_{2}$ population was grown at Jinghong in winter. Then, $204 \mathrm{~F}_{2}$ individuals were self-pollinated to generate $204 \mathrm{~F}_{2: 3}$ lines in conformity with QTL methods that used $\mathrm{F}_{2}$-derived $\mathrm{F}_{3}$ lines (Zhang and $\mathrm{Xu}$ 2004).

Field trials. Both Baoshan $\left(99^{\circ} 0^{\prime} \mathrm{N}, 24^{\circ} 55^{\prime} \mathrm{E}, 1,870 \mathrm{~m}\right.$ above sea level [masl]) and Dehong (24 $26^{\prime} \mathrm{N}, 98^{\circ} 35^{\prime} \mathrm{E}, 914$ masl) are hotspots for GLS in China and experience severe GLS epidemics almost every year. These two locations are optimal sites for natural inoculation of maize with C. zeae-maydis (Xu et al. 2014) and for germplasm evaluation for GLS resistance (Liu and Xu 2013; Wu et al. 2009; Xu et al. 2014; Zhang et al. 2012). In 2011 and 2012, parents, $F_{1}$, and the 204 $\mathrm{F}_{2: 3}$ lines were planted in a randomized complete-block design, with three replicates at each location. Plots were planted as single rows with a row spacing of $0.7 \mathrm{~m}$ and row length of $4.0 \mathrm{~m}$. Distance between two adjacent plants was $0.44 \mathrm{~m}$ and the population density was approximately 64,500 plants/ha. Trials were managed according to standard local practices.

GLS tests. The data of both plant response to the infection collected at day 28 after flowering and the flowering time were used for genetic analysis and QTL mapping. The evaluation of leaf infection with GLS followed the standard 1-to-9 scale reported by Saghai Maroof et al. (1993). The GLS resistance was evaluated using disease index and disease severity on a scale of 1 to 9 , where $1=$ highly resistant (none or few gray spots on leaves or lesion area being less than $5 \%$ of total leaf area), $3=$ resistant (a small number of spots on leaves or lesion area $=6$ to $10 \%$ of total leaf area), $5=$ moderately resistant (medium number of spots on leaves or lesion area $=11$ to $30 \%$ of total leaf area), 7 = susceptible (a large number of spots on leaves or lesion area $=31$ to $70 \%$ of total leaf area), and $9=$ highly susceptible (large lesion area on leaves or leaf death). Infected leaves corresponding to different categories are displayed in Figure 1. Date of anthesis (namely, date with pollen release in 50\% of plants in a row) was recorded as the flowering date and days from planting to anthesis were used for flowering time.

Analysis of GLS resistance and QTL genetic effect estimation. Analysis of variance (ANOVA) for resistance was conducted via PROC GLM of SAS 9.1 software (SAS Institute Inc. 2004). The joint segregation analysis of $\mathrm{P}_{1}, \mathrm{P}_{2}, \mathrm{~F}_{1}$, and $\mathrm{F}_{2: 3}$ with a mixed major gene plus polygene model was used to analyze the GLS resistance of the YML $32 \times$ Ye 478 maize population (Zhang and Xu 2004). This model had five subgenetic models: one pair of major genes (A model), two pairs of major genes (B model), polygenes ( $\mathrm{C}$ model), one pair of major genes plus polygenes (D model), and two pairs of major genes plus polygenes (E model). The assumptions for the A, B, C, D, and E models were as follows: diploid nuclear inheritance with no maternal or cytoplasmic effects; no interaction or linkage between major genes and polygenes, and no selection; the genetic effect of polygenes and the effect of the environment in any segregating population followed a normal distribution; and variances within the $\mathrm{P}_{1}, \mathrm{P}_{2}$, and $\mathrm{F}_{1}$ populations were equal. Based on these assumptions, 24 subgenetic models, in total, from the five genetic models involving additive, dominance, equal, epistasis, and negative effect were established (Wang and Gai 2001).

The generation parameters and component distributions were estimated with the maximum-likelihood method and iterated expectation and conditional maximization (IECM) algorithm (Dempster et al. 1977; McLachlan and Basford 1988; Wang and Gai 2001; Zhang and $\mathrm{Xu}$ 2004; Zhang et al. 2003). In the joint segregation analysis of the mixed genetic model, Akaike information criterion (AIC) was employed to determine which submodel best fit the data. A goodness-of-fit test was used to determine whether or not the selected model sufficiently explained the data. The best-fitting genetic model was selected according to both AIC and the results from goodness-offit tests (homogeneity test $U_{1}^{2}, U_{2}^{2}$, and $U_{3}^{2}$; Smirnov $\left[{ }_{n} W^{2}\right]$ test; and Kolmogorov $\left[D^{n}\right]$ test). From AIC, the models with the lowest AIC

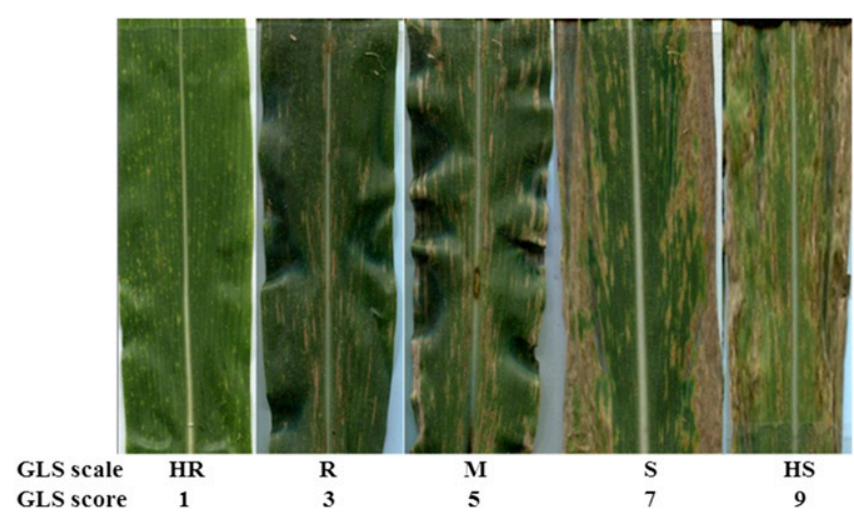

Fig. 1. Leaves infected by Cercospora zeae-maydis corresponding to different scales and grades. $H R=$ highly resistant to gray leaf spot $(G L S), R=$ resistant to $G L S, M=$ moderately resistant to GLS, $\mathrm{S}=$ susceptible to $\mathrm{GLS}$, and $\mathrm{HS}=$ highly susceptible to GLS. 
value or the relatively lower AIC value were chosen as the best-fit models. According to the test, the model with the lowest number of statistics among the best-fit models was selected as the best-fit model.

The genetic variances were estimated via the least-squares method according to the optimal genetic model (Gai and Wang 1998). In the mixed inheritance model, the phenotypic value $(p)$ can be expressed as the summation of population mean $(m)$, major gene effect $(g)$, polygene effect $(c)$, and environmental effect (e); that is, $p=m+g+$ $c+e$, where $g$ varies with different major genotypes and $c$ and $e$ are normally distributed variables. Based on this model, the phenotypic variation $\left(\sigma_{\mathrm{p}}^{2}\right)$ can be expressed as major gene variation $\left(\sigma_{\mathrm{mg}}^{2}\right)$, polygenic variation $\left(\sigma_{\mathrm{pg}}^{2}\right)$, and environmental variation $\left(\sigma_{\mathrm{e}}^{2}\right) \cdot \sigma_{\mathrm{p}}^{2}=\sigma_{\mathrm{mg}}^{2}+\sigma_{\mathrm{pg}}^{2}+\sigma_{\mathrm{e}}^{2}$; heritability of major gene $\left(\mathrm{h}_{\mathrm{mg}}^{2}\right)$ and heritability of polygene $\left(\mathrm{h}_{\mathrm{pg}}^{2}\right)$ were calculated, respectively, as

$$
\begin{aligned}
& \mathrm{h}_{\mathrm{mg}}^{2}(\%)=\left(\sigma_{\mathrm{mg}}^{2} / \sigma_{\mathrm{p}}^{2}\right) \times 100 \\
& \mathrm{~h}_{\mathrm{pg}}^{2}(\%)=\left(\sigma_{\mathrm{pg}}^{2} / \sigma_{\mathrm{p}}^{2}\right) \times 100
\end{aligned}
$$

Genotyping. Based on GLS resistance data at various sites in both 2011 and 2012, equal amounts of DNA from the five most susceptible lines were mixed to form the susceptible bulk and equal amounts of DNA from the five most resistant lines were mixed to form the resistant bulk for screening the polymorphic marker. In total, 760 simple-sequence repeats (SSR) were retrieved from the Maize Genetics and Genomics Database (http://www.maizegdb.org/) and synthesized by Sangon Biotech (Shanghai, China). In total, 167 polymorphic SSR markers (21.9\%) between two parents were used for genotyping the 204 individuals of the $F_{2}$ population, which were selfed to generate $\mathrm{F}_{2: 3}$ lines for phenotyping. A modified polymerase chain reaction (PCR) protocol according to McCouch et al. (2002) was used. The protocol consisted of a total volume of $10 \mu \mathrm{l}$ containing $20 \mathrm{ng}$ of genomic DNA, $0.3 \mu \mathrm{M}$ each primer, $1.0 \mu \mathrm{l}$ of $10 \times$ Taq DNA polymerase buffer (20 mM MgCl 2$), 0.1 \mathrm{mM}$ each dNTP (TransGen Biotech), and $1 \mathrm{U}$ of Taq DNA polymerase (TransGen Biotech). The reaction profile with Mastercycler gradient (Eppendorf) was $5 \mathrm{~min}$ at $94^{\circ} \mathrm{C}$; with 34 cycles of $45 \mathrm{~s}$ at $95^{\circ} \mathrm{C}, 45 \mathrm{~s}$ at $57^{\circ} \mathrm{C}$ annealing, and $1 \mathrm{~min}$ at $72^{\circ} \mathrm{C}$; and $10 \mathrm{~min}$ at $72^{\circ} \mathrm{C}$ for final extension. The PCR products were separated via electrophoresis on $6 \%$ polyacrylamide gel.

Table 1. Analysis of variances for gray leaf spot (GLS) resistance ${ }^{a}$

\begin{tabular}{lrrrrc}
\hline Source & DF & \multicolumn{1}{c}{ SS } & \multicolumn{1}{c}{ MS } & $\boldsymbol{F}$ value & Pr $>\boldsymbol{F}$ \\
\hline Lines & 203 & 6674.37 & 32.88 & 33.34 & $<0.0001^{* *}$ \\
Location & 1 & 1165.34 & 1165.34 & 1193.42 & $0.0007^{* *}$ \\
Year & 1 & 506.92 & 506.92 & 510.44 & 0.0623 \\
Repeat & 2 & 14.19 & 7.09 & 7.27 & $0.0086^{* *}$ \\
Lines $\times$ location & 203 & 475.69 & 2.34 & 2.38 & $0.0232^{*}$ \\
Lines $\times$ year & 203 & 150.71 & 0.74 & 0.77 & 0.0854 \\
Location $\times$ year & 1 & 177.34 & 177.34 & 181.61 & 0.4587 \\
Error & 1837 & 1793.77 & 0.98 & $\ldots$ & $\ldots$ \\
\hline
\end{tabular}

${ }^{\mathrm{a}} \mathrm{DF}=$ degree of freedom, $\mathrm{SS}=$ sum of squares, $\mathrm{MS}=$ mean squares, and $\mathrm{Pr}=$ probability; * and ** indicate significance at 5 and $1 \%$ probability levels, respectively.
Map construction and QTL detection. A linkage map was constructed by use of IciMapping v3.1 software with polymorphic SSR markers between two parents (Wang et al. 2011). Linkage groups were identified using the Group command to identify linkage groups with a logarithm of odds (LOD) score of 3.0, and recombination frequency was converted into centimorgans using the Kosambi mapping function (Kosambi 1943). The QTL detection was performed with Breeding View in IB Workflow System (https://www.integratedbreeding.net/ breeding-management-system) by inclusive composite-interval mapping (CIM) and the results were verified by the IciMapping v3.1 software with ICIM-ADD mapping method (Wang et al. 2011). A LOD of 3.0 was used to declare a QTL as significant. The percentages of phenotypic variance explained $\left(R^{2}\right)$ by individual QTL and additive effects at LOD peaks were obtained through stepwise regression. Adjacent QTL on the same chromosome were considered different when the distances between the curve peaks were $>20$ centimorgans $(\mathrm{cM})$. The QTL overlapping within intervals of $20 \mathrm{cM}$ on the same chromosome at both locations were considered to be the same. The CIM method was used to detect QTL for the quantitative traits because of its greater efficiency of QTL detection. This method uses stepwise regression for identifying the significant flanking markers, one-way scanning to identify additive effect, and two-way scanning to identify digenic-epistatic effect (Murray et al. 2013).

\section{Results}

Phenotypic analysis of GLS response and flowering time. Results from both locations (Baoshan and Dehong) in the 2 years (2011 and 2012) showed that resistant parental line YML 32 expressed either resistance or high resistance, with disease scores of 1 to 3, whereas susceptible parental line Ye 478 was highly susceptible, with disease scores of 7 to 9. Because ANOVA (Table 1) showed that there were no significant differences in GLS disease scores between 2011 and 2012, average GLS disease scores from both years were used for the rest of the analyses.

The GLS disease scores showed wide segregation among $\mathrm{F}_{2: 3}$ lines and the average GLS disease score (3.76) and flowering time (79 days) at both locations were close to the midparent value (Table 2). The correlation coefficient of GLS disease scores and flowering time were $-0.82(P<0.01)$ and $-0.85(P<0.01)$ at Baoshan and Dehong, respectively. The results indicated that flowering time is strongly correlated with GLS resistance in this population. The frequency distribution of GLS disease scores and flowering time of the $\mathrm{F}_{2: 3}$ lines at the two locations showed continuous variation, confirming quantitative inheritance for GLS resistance (Fig. 2). The GLS disease scores and flowering time presented positive and negative skewness at both locations, indicating that complementary gene interactions might condition GLS resistance, with dominance genes playing an important role (Jayaramachandran et al. 2010; Samak et al. 2011). This means that intensive selection for GLS resistance in segregating generations would ensure rapid gains but, on the other hand, gains would be slow if mild selection is practiced.

Estimation of heritability for resistance to GLS. Statistical analysis of the average GLS resistance ratings for $204 \mathrm{~F}_{2: 3}$ lines across years and locations indicated the presence of significant differences among genotypes and between locations (Table 1). The

\begin{tabular}{|c|c|c|c|c|c|c|c|}
\hline \multirow[b]{2}{*}{ Location } & \multirow[b]{2}{*}{ Trait $^{\mathbf{a}}$} & \multicolumn{2}{|c|}{ Parent } & \multicolumn{4}{|c|}{$\mathbf{F}_{2: 3}$ line } \\
\hline & & YML 32 & Ye 478 & Average \pm SD $^{b}$ & Range & Skewness & Kurtosis \\
\hline \multirow[t]{2}{*}{ Baoshan } & GLS & 1.00 & 8.00 & $4.65 \pm 1.89$ & $1.00-8.89$ & 0.16 & -0.84 \\
\hline & FT & 79 & 71 & $75.00 \pm 2.07$ & $70.00-80.00$ & -0.29 & -0.56 \\
\hline \multirow[t]{2}{*}{ Dehong } & GLS & 1.00 & 9.00 & $2.87 \pm 1.59$ & $1.00-7.20$ & 0.95 & 0.06 \\
\hline & FT & 86 & 79 & $83.09 \pm 1.68$ & $79.00-86.00$ & -0.75 & -0.12 \\
\hline \multirow[t]{2}{*}{ Average } & GLS & 1.00 & 8.50 & $3.76 \pm 1.65$ & $1.00-7.95$ & 0.53 & -0.51 \\
\hline & FT & 83 & 75 & $79.16 \pm 1.74$ & $74.50-82.50$ & -0.51 & -0.51 \\
\hline
\end{tabular}

Table 2. Phenotypes of gray leaf spot (GLS) disease score and flowering time in the YML $32 \times$ Ye 478 lines and the parents in Baoshan and Dehong locations

a GLS $=$ the disease scores of gray leaf spot and FT $=$ the flowering time.

b $\mathrm{SD}=$ standard deviation. 
maximum-likelihood estimators and AIC values of five types of genetic models and 24 submodels (Table 3), including one major gene (A), two major genes (B), polygenes (C), one major gene plus polygene (D), and two major genes plus polygenes (E), were acquired by IECM arithmetic to jointly analyze the GLS resistance of the four generations (i.e., $\mathrm{P}_{1}, \mathrm{P}_{2}, \mathrm{~F}_{1}$, and $\mathrm{F}_{2: 3}$ ) from the $\mathrm{YML} 32 \times \mathrm{Ye} 478$ population using the mixed major gene plus polygene inheritance model (Gai and Wang 1998). A set of goodness-of-fit tests $\left(U_{1}^{2}, U_{2}^{2}, U_{3}^{2},{ }_{\mathrm{n}} W^{2}\right.$, and $D^{n}$ ) were further conducted for selecting an optimal model; the genetic models with relatively low AIC values are given in Table 4. According to the AIC criterion, model MX2-AD-AD was the optimal genetic model. This model has a smaller AIC value than those of the other 23 subgenetic models. The $F_{1}$ reaches 0.01 and 0.05 significance levels according to $U_{3}^{2}$ and ${ }_{\mathrm{n}} W^{2}$ tests, and $\mathrm{F}_{2: 3}$ reaches 0.05 significance levels based on the $U_{3}^{2}$ test. This indicated that the GLS resistance of maize fitted the MX2-AD-AD genetic model; namely, with two additive-dominance effect major genes plus an additive-dominance effect polygene. Broad-sense heritability for GLS resistance was estimated to be as high as 0.926 using the least-squares method based on the optimal genetic model (Table 3).

Construction of the linkage map. The $\mathrm{F}_{2: 3}$ lines derived from YML $32 \times$ Ye 478 were used to construct a linkage map. The linkage map was constructed using ICIM (Wang et al. 2011), including 10 linkage groups, and spanned a total of $3,614.3 \mathrm{cM}$ in genome, with an average of $10.0 \mathrm{cM}$ between every two adjacent markers.
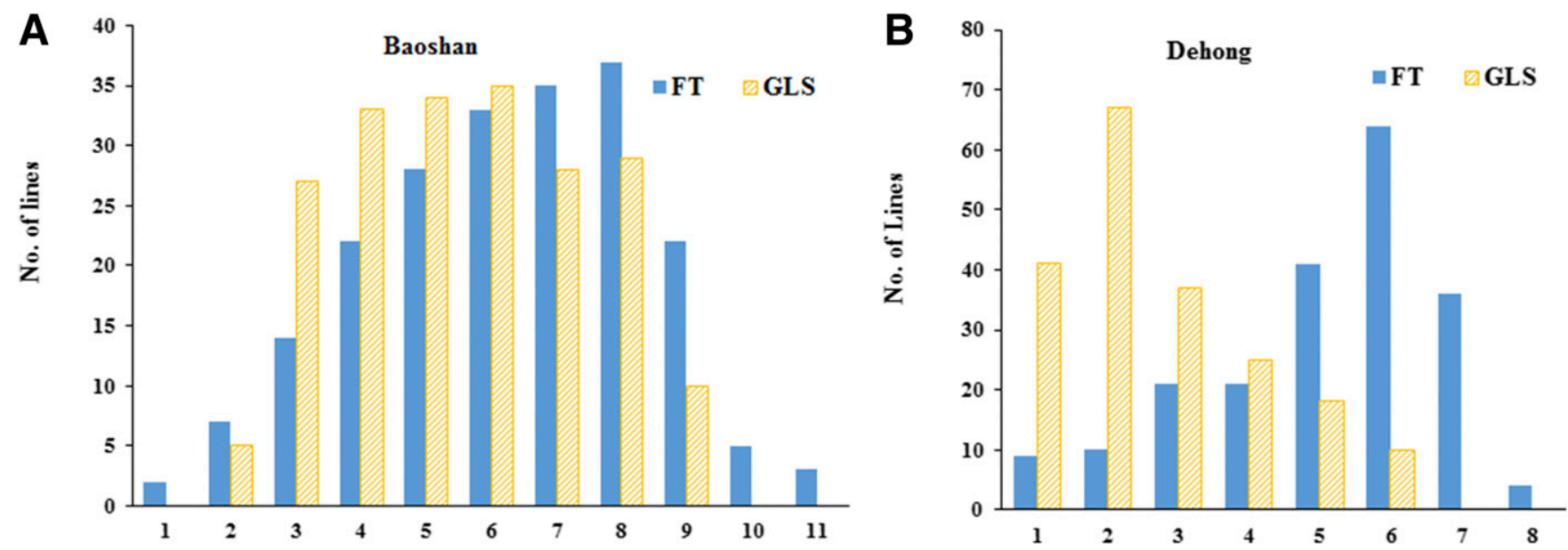

Fig. 2. Distribution of gray leaf spot (GLS) disease scores and flowering time in the $F_{2: 3}$ population. $G L S=G L S$ disease scores and FT = flowering time. A, Distribution of GLS disease scores and flowering time in the $F_{2: 3}$ population in Baoshan and $B$, distribution of GLS disease scores and flowering time in the $F_{2: 3}$ population in Dehong.

Table 3. Maximum log-likelihood (MLV) and Akaike's information criterion (AIC) values in various genetic models of four generations for gray leaf spot (GLS) resistance ${ }^{\mathrm{a}}$

\begin{tabular}{|c|c|c|c|c|c|c|}
\hline \multirow[b]{2}{*}{ Model $^{b}$} & \multirow[b]{2}{*}{ MLV } & \multirow[b]{2}{*}{ AIC } & \multicolumn{2}{|c|}{ Variation } & \multicolumn{2}{|c|}{ Heritability } \\
\hline & & & Major gene & Polygene & Major gene & Polygene \\
\hline $1 \mathrm{MG}-\mathrm{AD}$ & -400.2 & 812.5 & 1.589 & $\ldots$ & 0.583 & $\ldots$ \\
\hline $1 \mathrm{MG}-\mathrm{A}$ & -411.5 & 832.9 & 1.226 & $\ldots$ & 0.449 & $\ldots$ \\
\hline 1MG-EAD & -400.9 & 811.8 & 1.512 & $\ldots$ & 0.554 & $\ldots$ \\
\hline 1MG-AEND & -416.0 & 841.9 & 0.000 & $\ldots$ & 0.000 & $\ldots$ \\
\hline 2MG-ADI & -392.5 & 806.9 & 2.319 & $\ldots$ & 0.850 & $\ldots$ \\
\hline 2MG-AD & -392.9 & 799.9 & 2.214 & $\ldots$ & 0.811 & $\ldots$ \\
\hline 2MG-A & -405.6 & 821.2 & 1.629 & $\ldots$ & 0.597 & $\ldots$ \\
\hline 2MG-EA & -405.5 & 819.1 & 1.626 & $\ldots$ & 0.596 & $\ldots$ \\
\hline 2MG-AED & -397.9 & 805.8 & 1.869 & $\ldots$ & 0.685 & $\ldots$ \\
\hline 2MG-EEAD & -397.7 & 803.4 & 1.818 & $\ldots$ & 0.667 & $\ldots$ \\
\hline PG-ADI & -399.4 & 810.8 & $\ldots$ & 2.487 & $\ldots$ & 0.912 \\
\hline PG-AD & -399.4 & 808.9 & $\ldots$ & 2.486 & $\ldots$ & 0.911 \\
\hline MX1-AD-ADI & -386.8 & 789.7 & 1.667 & 0.820 & 0.611 & 0.301 \\
\hline MX1-AD-AD & -410.0 & 834.1 & 1.705 & 0.783 & 0.625 & 0.287 \\
\hline MX1-A-AD & -394.7 & 801.4 & 2.012 & 0.445 & 0.738 & 0.163 \\
\hline MX1-EAD-AD & -399.4 & 810.9 & 0.019 & 2.467 & 0.007 & 0.904 \\
\hline MX1-AEND-AD & -399.4 & 810.9 & 0.019 & 2.467 & 0.007 & 0.904 \\
\hline MX2-ADI-ADI & -378.4 & 780.8 & 2.554 & 0.000 & 0.936 & 0.000 \\
\hline MX2-ADI-AD & -380.1 & 778.2 & 2.547 & 0.000 & 0.934 & 0.000 \\
\hline MX2-AD-AD & -379.6 & 769.2 & 2.527 & 0.000 & 0.926 & 0.000 \\
\hline MX2-A-AD & -394.5 & 795.0 & 2.024 & 0.433 & 0.742 & 0.159 \\
\hline MX2-EAED-AD & -387.6 & 779.3 & 2.330 & 0.140 & 0.854 & 0.051 \\
\hline MX2-AED-AD & -399.4 & 804.9 & 0.017 & 2.470 & 0.006 & 0.905 \\
\hline MX2-EEAD-AD & -388.4 & 780.8 & 1.908 & 0.578 & 0.699 & 0.212 \\
\hline
\end{tabular}

a Symbol: ... indicates data not available.

b In these models, $1 \mathrm{MG}$ and 2MG represent one major gene and two major gene models, respectively; MX1 represents a mixed model with one major gene and polygene; MX2 indicates a mixed model with two major gene and polygene; PG represents polygene; and A, D, E, I, and N represent the genes with additive, dominance, equal, epistatic, and negative effects, respectively. For example, model MX2-AD-AD means a mixed model with two major genes of additive-dominance effects and additive-dominance polygene. 
Among the 167 polymorphic SSR markers (Supplementary Table S1) between the two parents, 17, 20, 22, 28, 19, 12, 13, 13, 15, and 8 markers were located on chromosomes $1,2,3,4,5,6,7,8,9$, and 10 , respectively. The linkage map is well established because the bins of markers used and listed as Supplementary Table S1 were evenly distributed on chromosomes compared with other IBM maps. Therefore, the linkage map was suitable for QTL detection.

QTL and their genetic effects for GLS resistance. Seven QTL related to GLS disease scores and six QTL corresponding to flowering time were detected from the population of YML $32 \times$ Ye 478, with different numbers of QTL being recorded at the two locations. At Baoshan, five QTL (qRgls.yaas-2, qRgls.yaas-3-1, qRgls.yaas-4, qRgls.yaas-5, and qRgls.yaas-8-1) for GLS disease scores were detected in bins 2.06, 3.04, 4.06, 5.04, and 8.04, respectively (Table 5; Fig. 3). These five QTL showed additive effects from 0.36 to 1.13 and explained 1.8 to $17.9 \%$ of the variation of GLS disease scores. In addition to additive effects, a dominance effect $(0.60)$ was also found for QTL qRgls.yaas-5, which explained $1.8 \%$ of the variation of GLS disease scores. As for the flowering time, three QTL (qFt.yaas-3, qFt.yaas-4, and qFt.yaas-8) were detected in bins 3.05, 4.06, and 8.04, respectively (Table 6). These three QTL showed additive effects from 0.71 to 0.99 and explained 5.92 to $11.48 \%$ of the variation of flowering time. In addition to additive effects, a dominance effect (0.56) was also found for QTL qFt.yaas-8. Two of the three QTL for flowering time were overlapped with the QTL for

Table 4. Tests of gray leaf spot (GLS) resistance for goodness-of-fit of genetic models with lower Akaike's information criterion (AIC) values in the cross of YML $32 \times$ Ye $478^{\mathrm{a}}$

\begin{tabular}{|c|c|c|c|c|c|}
\hline Model, population' & $U_{1}^{2}$ & $U_{2}^{2}$ & $U_{3}^{2}$ & ${ }_{n} W^{2}$ & $D^{n}$ \\
\hline \multicolumn{6}{|l|}{ MX1-AD-ADI } \\
\hline $\mathrm{P}_{1}$ & 0.0000 & 0.1562 & 2.5000 & 0.1667 & 0.5000 \\
\hline $\mathrm{F}_{1}$ & 0.0000 & 0.3616 & $5.7851 *$ & 0.1285 & 0.4584 \\
\hline $\mathrm{P}_{2}$ & 0.0000 & 0.1562 & 2.5000 & 0.1667 & 0.5000 \\
\hline $\mathrm{F}_{2: 3}$ & 0.0152 & 0.0055 & 0.6008 & 0.0703 & 0.0065 \\
\hline \multicolumn{6}{|l|}{ MX2-ADI-ADI } \\
\hline $\mathrm{P}_{1}$ & 0.0000 & 0.1562 & 2.5000 & 0.1667 & 0.5000 \\
\hline $\mathrm{F}_{1}$ & 0.0000 & 0.4819 & $7.7097 * *$ & 0.1468 & 0.4792 \\
\hline $\mathrm{P}_{2}$ & 0.0000 & 0.1562 & 2.5000 & 0.1667 & 0.5000 \\
\hline $\mathrm{F}_{2: 3}$ & 0.0002 & 0.0010 & 0.0046 & 0.0167 & 0.0039 \\
\hline \multicolumn{6}{|l|}{ MX2-ADI-AD } \\
\hline $\mathrm{P}_{1}$ & 0.6602 & 1.1057 & 1.1220 & 0.2217 & 0.6659 \\
\hline $\mathrm{F}_{1}$ & 0.0941 & 0.0505 & $4.3558 *$ & 0.1181 & 0.3726 \\
\hline $\mathrm{P}_{2}$ & 0.6602 & 1.1057 & 1.1220 & 0.2217 & 0.6659 \\
\hline $\mathrm{F}_{2: 3}$ & 0.0047 & 0.0070 & 0.0048 & 0.0178 & 0.0032 \\
\hline \multicolumn{6}{|l|}{$M X 2-A D-A D$} \\
\hline $\mathrm{P}_{1}$ & 0.0308 & 0.0481 & 2.4236 & 0.1692 & 0.4642 \\
\hline $\mathrm{F}_{1}$ & 0.0035 & 0.4978 & $6.7214 * *$ & $0.1378^{*}$ & 0.4810 \\
\hline $\mathrm{P}_{2}$ & 0.0308 & 0.0481 & 2.4236 & 0.1692 & 0.4642 \\
\hline $\mathrm{F}_{2: 3}$ & 0.0033 & 0.0007 & $0.0129 *$ & 0.0240 & 0.0031 \\
\hline \multicolumn{6}{|l|}{ MX2-EAED-AD } \\
\hline $\mathrm{P}_{1}$ & 0.1169 & 0.4946 & 2.2162 & 0.1764 & 0.5698 \\
\hline $\mathrm{F}_{1}$ & 0.0311 & 0.1357 & $4.6526^{*}$ & 0.1183 & 0.4064 \\
\hline $\mathrm{P}_{2}$ & 0.1169 & 0.4946 & 2.2162 & 0.1764 & 0.5698 \\
\hline $\mathrm{F}_{2: 3}$ & 0.0962 & 0.0484 & 0.1034 & 0.0713 & 0.0042 \\
\hline \multicolumn{6}{|l|}{ MX2-EEAD-AD } \\
\hline $\mathrm{P}_{1}$ & 0.0051 & 0.2148 & 2.4872 & 0.1671 & 0.5146 \\
\hline $\mathrm{F}_{1}$ & 0.0010 & 0.3215 & $5.7254 *$ & 0.1280 & 0.4510 \\
\hline $\mathrm{P}_{2}$ & 0.0051 & 0.2148 & 2.4872 & 0.1671 & 0.5146 \\
\hline $\mathrm{F}_{2: 3}$ & 0.0204 & 0.0000 & 0.2834 & 0.0540 & 0.0114 \\
\hline
\end{tabular}

${ }^{\text {a }} U_{1}^{2}, U_{2}^{2}$, and $U_{2}^{2}=$ homogeneity tests, ${ }_{\mathrm{n}} W^{2}=$ Smirnov test, and $D^{n}=$ Kolmogorov test; * and ** indicate significance at 5 and $1 \%$ probability levels, respectively.

b In these models, MX1 represents a mixed model with one major gene and polygene; MX2 indicates a mixed model with two major gene and polygene; and $\mathrm{A}, \mathrm{D}, \mathrm{E}$, and I represent the genes with additive, dominance, equal, epistatic, and negative effects, respectively. For example, model MX2AD-AD means a mixed model with two major genes of additive-dominance effects and additive-dominance polygene.
GLS disease scores, which indicated that these two QTL (qRgls. yaas-4/qFt.yaas-4, and qRgls.yaas-8-1/qFt.yaas-8) were likely at the same chromosome location and corresponded to both GLS resistance and flowering time.

At Dehong, four QTL for resistance to GLS (qRgls.yaas-3-2, qRgls.yaas-4, qRgls.yaas-8-1, and qRgls.yaas-8-2) were identified in bins of 3.05, 4.06, 8.04, and 8.01, respectively (Table 5; Fig. 3). These four QTL accounted for 1.8 to $18.1 \%$ of the variation of GLS disease scores. The additive effects of these QTL were 0.30 to 0.96. Similar to Baoshan, the QTL qRgls.yaas-3-2 also showed a dominance effect of 0.43 , explaining $1.8 \%$ of the variation of GLS disease scores. As for the flowering time, four QTL ( $q F t$. yaas-2-1, qFt.yaas-4, qFt.yaas-5, and qFt.yaas-8) were identified in bins of 2.06, 4.06, 5.04, and 8.04, respectively (Table 6). The QTL (qFt.yaas-2-1 and qFt.yaas-5) for flowering time were not overlapped with the QTL for the GLS disease scores. These four QTL accounted for 0.15 to $21.42 \%$ of the variation of flowering time. The additive effects of these QTL were 0.05 to 1.10 . The QTL qFt. yaas-5 showed a dominance effect of 0.59. The result from Dehong location further showed that the two QTL (qRgls.yaas-4/qFt.yaas-4 and qRgls.yaas-8-1/qFt.yaas-8) were likely at the same chromosome and corresponded to both GLS resistance and flowering time. However, the two QTL for GLS disease scores ( $q$ Rgls.yaas-3-2 and qRgls.yaas-8-1) did not associate with flowering time, and the QTL qFt.yaas-2-1 for flowering time did not corresponded to GLS resistance.

When the average value of GLS disease scores was used from the two locations across 2 years, five QTL (namely, $q$ Rgls.yaas-2, $q R g l s$. yaas-3-2, qRgls.yaas-4, qRgls.yaas-5, and qRgls.yaas-8-1) were detected in bins 2.06, 3.05, 4.06, 5.04, and 8.04, respectively (Table 5; Fig. 3). As for the flowering time, four QTL (namely, qFt.yaas-2-2, qFt.yaas-3, qFt.yaas-4, and qFt.yaas-8) were detected in bins 2.06, 3.05, 4.06, and 8.04, respectively (Table 6). These four QTL for flowering time overlapped the QTL for the GLS disease scores, which indicated that the four (qRgls.yaas-2/qFt.yaas-2-2, qRgls.yaas-3-2/qFt.yaas-3, qRgls.yaas-4/qFt.yaas-4, and qRgls.yaas8-1/qFt.yaas-8) were likely at the same chromosome locations and corresponded to both GLS resistance and flowering time. The results from averages seemed to detect most of the QTL in different locations, suggesting that multiple locations and multiple tests would give more reliable results.

By examining sources of the GLS resistance, we found that the QTL $q$ Rgls.yaas-8-1 and qFt.yaas-8, with the largest additive effect, were derived from the resistant parent YML 32, suggesting that the tropical line YML 32 was a good source for resistance to GLS, while there were five resistance QTL alleles identified to be originated from the susceptible parent Ye 478 (Tables 5 and 6). Comparing results from both locations, we found that qRgls.yaas-8-1 and qFt.yaas- 8 had the largest effect, which was consistent at both locations. These results further confirmed that there was an important QTL for resistance to GLS from the resistant parent YML 32, as found in our previous study (Zhang et al. 2012). Thus, YML 32 derived from the well-known maize germplasm Suwan 1 could serve as a good source of resistance to GLS in breeding programs. Among the QTL for the GLS disease scores and flowering time, only two QTL ( $q$ Rgls.yaas4/qFt.yaas-4 and qRgls.yaas-8-1/qFt.yaas-8) were identified at both locations. The fact that different QTL were detected at the two locations implied that environmental factors or experimental errors might have affected QTL detection, especially the QTL with smaller effects.

The two QTL qRgls.yaas-5 (bin 5.04) and qRgls.yaas-8-1 (bin 8.04) for GLS disease scores identified from YML 32 were not identified in the consensus GLS resistance QTL in bins 5.03 and 8.06 in the previous studies (Shi et al. 2007). Among the five QTL ( $q$ Rgls. yaas-2, qRgls.yaas-3-1, qRgls.yaas-3-2, qRgls.yaas-4, and qRgls. yaas-8-2) for GLS disease scores identified from Ye 478, qRgls. yaas-3-2 (bin 3.05) and qRgls.yaas-8-2 (bin 8.01) were also not located in the consensus GLS resistance QTL in bins 3.04, and 8.06 in previous studies (Shi et al. 2007). These results indicated that qRgls.yaas-3-2, qRgls.yaas-5, qRgls.yaas-8-1, and qRgls.yaas-8-2 
Chromosome 1

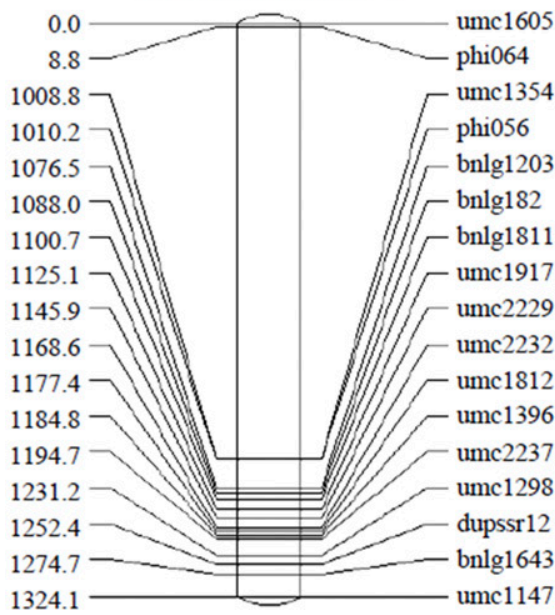

\section{Chromosome 4}

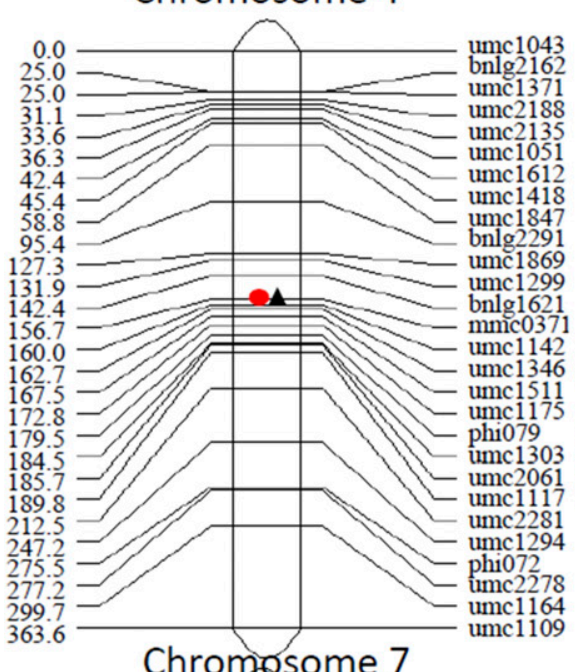
Chromosome 7

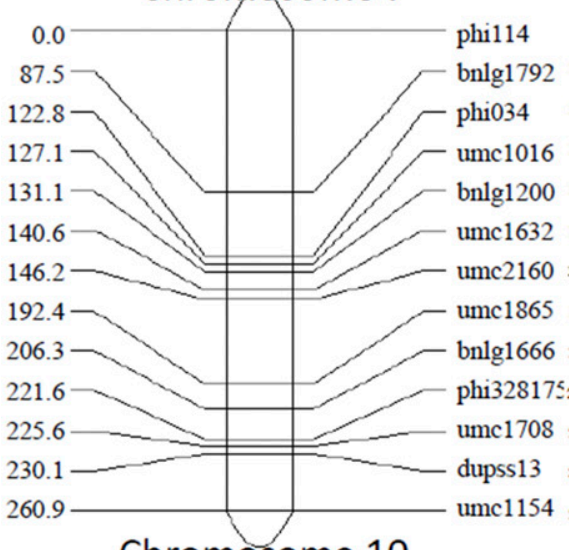

\section{Chromosome 10}

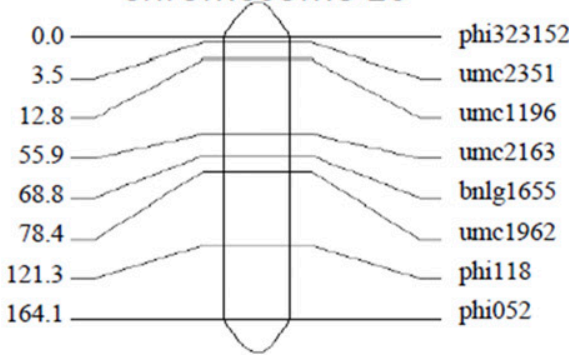

Chromosome 2

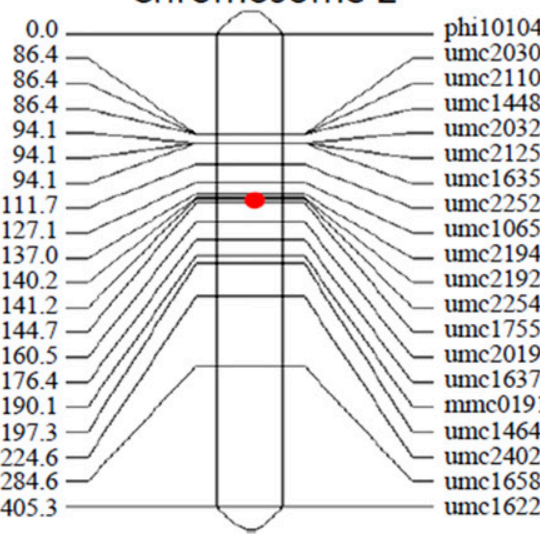

\section{Chromosome 5}

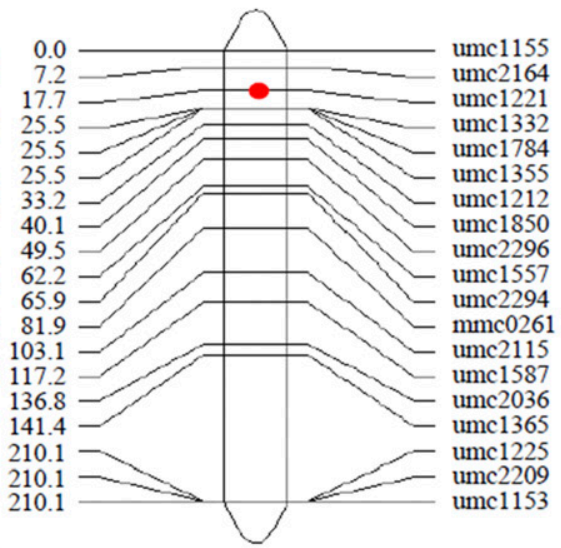

Chromosome 8

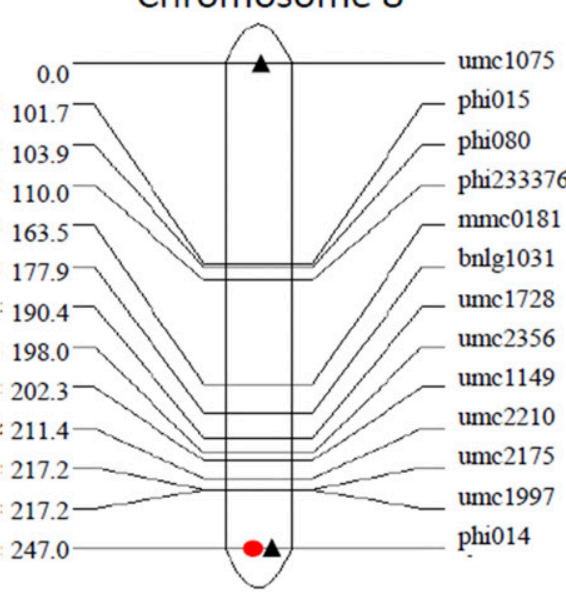

Chromosome 3
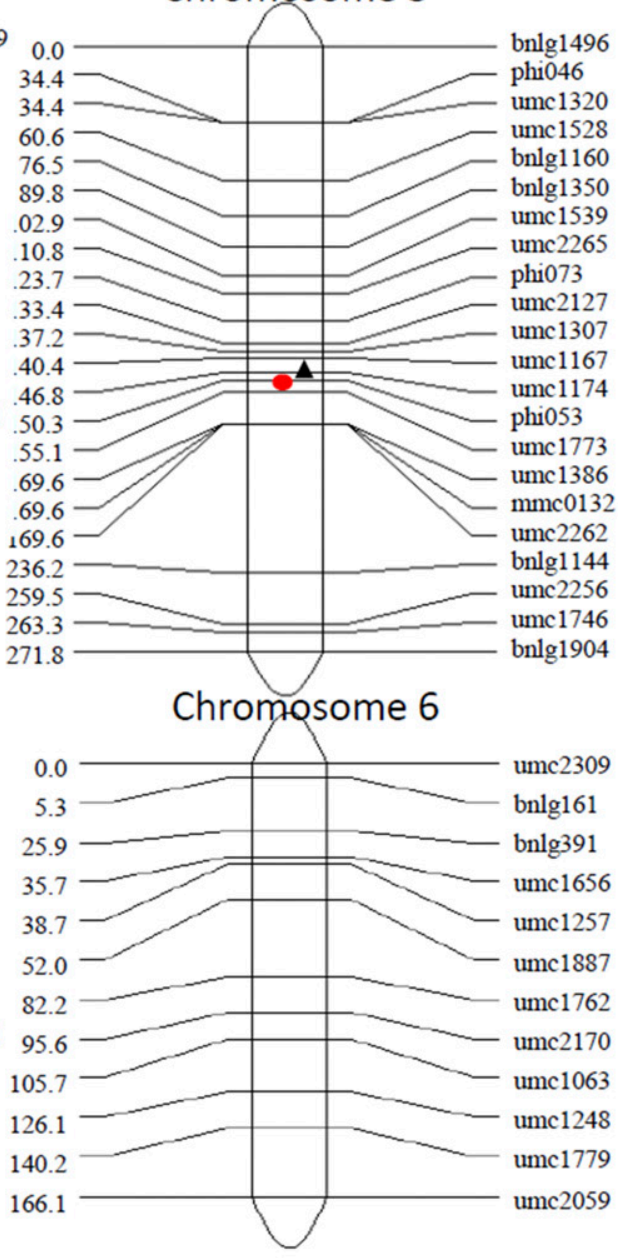

\section{Chromosome 9}

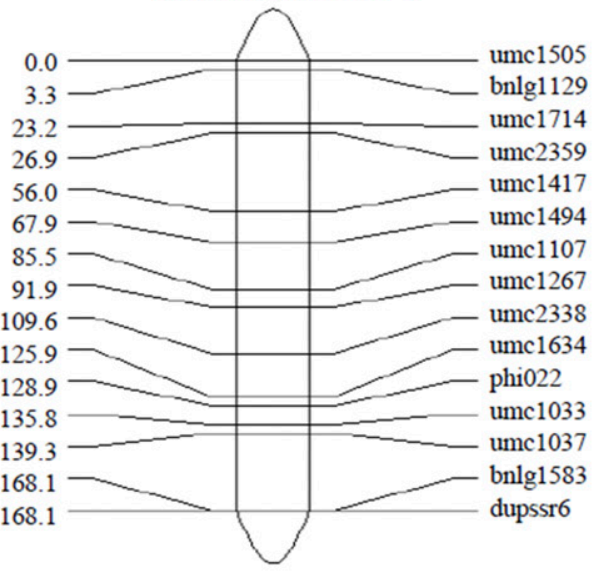

- QTL identified at Baoshan - QTL identified at Dehong

Fig. 3. Positions of quantitative trait loci (QTL) for gray leaf spot resistance in Baoshan and Dehong locations. 
might be new GLS resistance source for use in maize breeding program.

\section{Discussion}

Elwinger et al. (1990) had suggested that dominance should be included in models to fully explain the inheritance of GLS resistance. The results from our study offered strong evidence that dominanceeffect QTL played a role in controlling resistance to GLS (Table 5). In addition, the results from the joint segregation analysis in this study suggested that GLS resistance fitted the MX2-AD-AD genetic model. This result further suggested that additive-dominance effects were a major component of GLS resistance and that there might be two major genes and polygenes controlling GLS resistance in the YML $32 \times$ Ye 478 population. Both joint segregation genetic analysis and QTL analysis suggested that both additive and dominance effects existed in YML $32 \times$ Ye 478, which agreed with the findings of Elwinger et al. (1990) that GLS resistance was controlled by major genes with additive effects and polygenes with additive-dominance effects.

It is widely known that disease resistance can be inherited from a resistant parent. However, previous research also demonstrated that a highly susceptible parent also has the possibility of improving disease resistance in the offspring (Wang et al. 2012). Our previous study (Zhang et al. 2012) showed that a detected GLS resistance
QTL, qRgls3, was derived from the susceptible parent Q 11 and it could account for $10.73 \%$ of the variation for GLS resistance at Dehong. To confirm this finding, this study used a different susceptible parent (Ye 478) to cross with the same GLS-resistant parent (YML 32). The results showed that some QTL for resistance to GLS were derived from the susceptible parent Ye 478 in the YML $32 \times$ Ye 478 population. This further demonstrated that susceptible plants may also contain valuable resistance genes or QTL for improving disease resistance as reported in cotton (Wang et al. 2012), cacao (Crouzillat et al. 2000), pepper (Lefebvre and Palloix 1996), maize (Bubeck et al. 1993; Freymark et al. 1994), mung bean (Young et al. 1993), and tomato (Danesh et al. 1994). Therefore, in a maize breeding program, it is recommended that the background of the susceptible parent be carefully considered because of the potential of finding different QTL for resistance to GLS.

In a previous study (Zhang et al. 2012), a major QTL on chromosome 8 was found to confer GLS resistance in the YML $32 \times \mathrm{Q} 11$ population. In this study, the major QTL qRgls.yaas-8-1 and qFt. yaas -8 was detected on the same chromosome from the YML $32 \times$ Ye 478 population in both locations. These results further demonstrated that the inbred line YML 32 derived from Suwan 1 could be a valuable source of GLS resistance in maize breeding programs aimed at improving GLS resistance worldwide. It has all the advantages of a tropical maize germplasm such as high stress tolerance, a

Table 5. Quantitative trait loci (QTL) detected for gray leaf spot (GLS) disease scores in the $\mathrm{F}_{2: 3}$ population derived from the cross of YML $32 \times$ Ye $478^{\mathrm{a}}$

\begin{tabular}{|c|c|c|c|c|c|c|c|c|}
\hline Location, QTL & Bin & Flanking markers & Position (cM) & LOD & Additive effect & Dominant effect & Source of resistance & $R^{2}(\%)$ \\
\hline \multicolumn{9}{|l|}{ Baoshan } \\
\hline qRgls.yaas-2 & 2.06 & umc2192-umc1755 & 141.1 & 2.60 & 0.46 & - & Ye 478 & 2.9 \\
\hline qRgls.yaas $-3-1$ & 3.04 & umc1174-umc1773 & 152.0 & 6.96 & 0.80 & - & Ye 478 & 9.0 \\
\hline qRgls.yaas-4 & 4.06 & umc1142-bnlg1621 & 211.6 & 7.32 & 0.80 & - & Ye 478 & 9.1 \\
\hline qRgls.yaas-5 & 5.04 & umc2164-umc1332 & 17.5 & 3.00 & 0.36 & 0.60 & YML 32 & 1.8 \\
\hline qRgls.yaas-8-1 & 8.04 & umc1997-umc1505 & 248.2 & 11.49 & 1.13 & - & YML 32 & 17.9 \\
\hline \multicolumn{9}{|l|}{ Dehong } \\
\hline qRgls.yaas-3-2 & 3.05 & umc1167-phi053 & 148.6 & 2.26 & 0.30 & 0.43 & Ye 478 & 1.8 \\
\hline qRgls.yaas-4 & 4.06 & umc1142-bnlg1621 & 211.6 & 5.67 & 0.63 & - & Ye 478 & 7.8 \\
\hline qRgls.yaas-8-1 & 8.04 & umc1997-umc1505 & 248.2 & 10.60 & 0.96 & - & YML 32 & 18.1 \\
\hline qRgls.yaas $-8-2$ & 8.01 & umc1154-phi015 & 0.0 & 3.67 & 0.51 & - & Ye 478 & 5.1 \\
\hline \multicolumn{9}{|l|}{ Average } \\
\hline qRgls.yaas-2 & 2.06 & umc2192-umc1755 & 141.1 & 4.18 & 0.53 & - & Ye 478 & 5.1 \\
\hline qRgls.yaas $-3-2$ & 3.05 & umc1167-phi053 & 148.6 & 5.89 & 0.62 & - & Ye 478 & 7.1 \\
\hline qRgls.yaas -4 & 4.06 & umc1142-bnlg1621 & 211.6 & 6.52 & 0.65 & - & Ye 478 & 7.8 \\
\hline qRgls.yaas -5 & 5.04 & umc2164-umc1332 & 17.5 & 2.31 & 0.18 & 0.53 & YML 32 & 0.6 \\
\hline qRgls.yaas $-8-1$ & 8.04 & umc1997-umc1505 & 248.2 & 12.69 & 1.04 & - & YML 32 & 19.9 \\
\hline
\end{tabular}

${ }^{\mathrm{a}} \mathrm{Bin}=$ chromosome bin number, $\mathrm{cM}=$ centimorgans, LOD = logarithm of odds, Additive effect represents half the difference between the means of the homozygous classes at a given QTL, Dominance effect represents the difference between the mean of the heterozygous class and the mean of the homozygous classes at a given QTL, and - indicates no dominance effect on resistance.

Table 6. Quantitative trait loci (QTL) detected for flowering time in the $\mathrm{F}_{2: 3}$ population derived from the cross of YML $32 \times$ Ye $478^{\mathrm{a}}$

\begin{tabular}{|c|c|c|c|c|c|c|c|c|}
\hline Location, QTL & Bin & Flanking markers & Position (cM) & LOD & Additive effect & Dominant effect & Source of resistance & $R^{2}(\%)$ \\
\hline \multicolumn{9}{|l|}{ Baoshan } \\
\hline qFt.yaas-3 & 3.05 & umc1167-phi053 & 148.6 & 4.41 & 0.71 & - & Ye 478 & 5.92 \\
\hline qFt.yaas-4 & 4.06 & umc1142-bnlg1621 & 211.6 & 7.77 & 0.99 & - & Ye 478 & 11.48 \\
\hline qFt.yaas-8 & 8.04 & umc1997-umc1505 & 248.2 & 7.24 & 0.97 & 0.56 & YML 32 & 11.00 \\
\hline \multicolumn{9}{|l|}{ Dehong } \\
\hline qFt.yaas-2-1 & 2.06 & umc2194- umc2254 & 139.6 & 3.48 & 0.50 & - & Ye 478 & 4.46 \\
\hline qFt.yaas-4 & 4.06 & umc1142-bnlg1621 & 211.6 & 5.12 & 0.61 & - & Ye 478 & 6.58 \\
\hline qFt.yaas-5 & 5.04 & umc2164-umc1332 & 17.5 & 2.02 & 0.05 & 0.59 & Ye 478 & 0.15 \\
\hline qFt.yaas-8 & 8.04 & umc1997-umc1505 & 248.2 & 12.39 & 1.10 & - & YML32 & 21.42 \\
\hline \multicolumn{9}{|l|}{ Average } \\
\hline qFt.yaas-2-2 & 2.06 & umc2192-umc1755 & 141.1 & 4.38 & 0.58 & - & Ye 478 & 5.58 \\
\hline qFt.yaas-3 & 3.05 & umc1167-phi053 & 148.6 & 5.70 & 0.67 & - & Ye 478 & 7.39 \\
\hline qFt.yaas-4 & 4.06 & umc1142-bnlg1621 & 211.6 & 7.16 & 0.76 & - & Ye 478 & 9.34 \\
\hline qFt.yaas-8 & 8.04 & umc1997-umc1505 & 248.2 & 12.01 & 1.10 & - & YML 32 & 19.68 \\
\hline
\end{tabular}

${ }^{\mathrm{a}}$ Bin $=$ chromosome bin number, $\mathrm{cM}=$ centimorgans, LOD = logarithm of odds, Additive effect represents half the difference between the means of the homozygous classes at a given QTL, Dominance effect represents the difference between the mean of the heterozygous class and the mean of the homozygous classes at a given QTL, and - indicates no dominance effect on resistance. 
strong root system, and long stay green period, as compared with temperate maize germplasm, and can be widely utilized in FAO maize maturity zones of 700 and above. More importantly, all the released hybrids such as Yunrui 1, Yunrui 2, Yunde 3, and so on developed from the inbred line YML 32 possessed a high level of resistance to GLS. Maize breeders worldwide (Fan et al. 2014; Goodman 2004) have been trying to incorporate tropical maize germplasm into elite temperate maize germplasm to increase genetic variability for disease and pest resistance or yield. In our breeding program, we used YML 32 as the recurrent parent being crossed with temperate inbreds. We found that the progenies of YML 32 were not only highly resistant to GLS but also recovered most favorable traits such as yield, plant height, and so on from the temperate elite germplasm. This result not only confirmed that YML 32 was a very good source germplasm for GLS resistance but also further indicated that YML 32 had good combining ability with temperate germplasm for maize breeding aiming at improving disease resistance as well as keeping the most favorable traits of elite local germplasm.

Previous studies showed that GLS resistance correlated with flowering time (Balint-Kurti et al. 2008; Benson et al. 2015; Bubeck et al. 1993; Clements et al. 2000; Zwonitzeret al. 2010). In this study, the correlation coefficient of GLS disease scores and flowering were -0.82 and -0.85 at Baoshan and Dehong, respectively. Among all identified QTL, five QTL (qRgls.yaas-2/qFt.yaas-2-2, qRgls.yaas3-2/qFt.yaas-3, qRgls.yaas-4/qFt.yaas-4, qRgls.yaas-5/qFt.yaas-5, and qRgls.yaas-8-1/qFt.yaas-8) corresponded to GLS resistance and flowering time. These results further demonstrated that flowering time is strongly correlated with GLS resistance. However, the major QTL qRgls.yaas-8-1/qFt.yaas-8 was not the same as the QTL vgt1 (bin 8.05) for flowering time due to the different position on the chromosome (Salvi et al. 2007). Except for QTL qFt.yaas-2-1, identified on chromosome 2 at the Dehong location, all other QTL for flowering time were overlapped with the QTL for GLS disease scores. Therefore, the QTL for resistance to GLS are also QTL for flowering time and selection for flowering time is also selection for QTL to GLS resistance. However, Berger et al. (2014) thought that QTL for flowering time in the population of CML444 $\times$ SC Malawi did not correspond to QTL for GLS resistance. These contrary results might indicate either that the correlation between the flowering time and GLS resistance might vary from germplasm to germplasm or more markers may be needed to distinguish QTL based on GLS disease score and flowering time. Careful selection for maturity and disease resistance can be utilized in tandem for identifying new elite breeding germplasm adapted for the different maize maturity zones.

\section{Acknowledgments}

We thank S. Shao and B. Huang from Baoshan and Dehong Institute of Agricultural Sciences for their help in field management. The study was supported by the Yunnan Leading Talent Project (2014HA002), China 948 Project (2015Z73), National Natural Science Foundation of China Project (31060192 and 30960198), and Middle-age and Young Academic Leader and Cultivating Talent Project of Yunnan Province (2011CI037).

\section{Literature Cited}

Balestre, M., Von Pinho, R. G., and Brito, A. H. 2012. Bayesian inference to study genetic control of resistance to gray leaf spot in maize. Genet. Mol. Res. 11: 17-29.

Balint-Kurti, P. J., Wisser, R., and Zwonitzer, J. C. 2008. Use of an advanced intercross line population for precise mapping of quantitative trait loci for gray leaf spot resistance in maize. Crop Sci. 48:1696-1704.

Benson, J. M., Poland, J. A., Benson, B. M., Stromberg, E. L., and Nelson, R. J. 2015. Resistance to gray leaf spot of maize: Genetic architecture and mechanisms elucidated through nested association mapping and near-isogenic line analysis. PLoS Genet. 11:e1005045.

Berger, D. K., Carstens, M., Korsman, J. N., Middleton, F., Kloppers, F. J., Tongoona, P., and Myburg, A. A. 2014. Mapping QTL conferring resistance in maize to gray leaf spot disease caused by Cercospora zeina. BMC Genet. 15:60

Bubeck, D. M., Goodman, M. M., Beavis, W. D., and Grant, D. 1993. Quantitative trait loci controlling resistance to gray leaf spot in maize. Crop Sci. 33:838-847.

Chevin, L., and Hospital, F. 2008. Selective sweep at a quantitative trait locus in the presence of background genetic variation. Genetics 180:1645-1660.
Clements, M. J., Dudley, J. W., and White, D. G. 2000. Quantitative trait loci associated with resistance to gray leaf spot of corn. Phytopathology 90: 1018-1025.

Coates, S. T., and White, D. G. 1994. Sources of resistance to gray leaf spot of corn. Plant Dis. 78:1153-1155.

Crous, P. W., and Braun, U. 2003. Mycosphaerella and its anamorphs. 1. Names published in Cercospora and Passalora. CBS Biodivers. Ser. 1:1-571.

Crous, P. W., Groenewald, J. Z., Groenewald, M., Caldwell, P., Braun, U., and Harrington, T. C. 2006. Species of Cercospora associated with grey leaf spot of maize. Stud. Mycol. 55:189-197.

Crouzillat, D., Phillips, W., Fritz, P. J., and Pétiard, V. 2000. Quantitative trait loci analysis in Theobroma cacao L. using molecular markers. Inheritance of polygenic resistance to Phytophthora palmivora in two related cacao populations. Euphytica 114:25-36.

Dai, J. R., and E, L. Z. 2010. Scientific and technological innovation of maize breeding in China. J. Maize Sci. 18:1-5. (In Chinese with English abstract)

Danesh, D., Aarons, S., McGill, G. E., and Young, N. D. 1994. Genetic dissection of oligogenic resistance to bacterial wilt in tomato. Mol. Plant-Microbe Interact. 7:464-471.

Danson, J., Lagat, M., Kimani, M., and Kuria, A. 2008. Quantitative trait loci (QTLs) for resistance to gray leaf spot and common rust diseases of maize. Afr. J. Biotechnol. 7:3247-3254

Dempster, A. P., Laird, N. M., and Robin, D. B. 1977. Maximum likelihood from incomplete data via the EM algorithm. J. R. Stat. Soc. B 39:1-38.

Donahue, P. J., Stromberg, E. L., and Myers, S. L. 1991. Inheritance of reaction to gray leaf spot in a diallel cross of 14 maize inbreds. Crop Sci. 31:926-931.

Elwinger, G. F., Johnson, M. W., Hill, R. R., Jr., and Ayers, J. E. 1990. Inheritance of resistance to gray leaf spot of maize. Crop Sci. 30:350-358.

Fan, X. M., Zhang, Y. D., Yao, W. H., Bi, Y. Q., Liu, L., Chen, H. M., and Kang, M. S. 2014. Reciprocal diallel crosses impact combining ability, variance estimation, and heterotic group classification. Crop Sci. 54:89-97.

Freymark, P. J., Lee, M., Martinson, C. A., and Woodman, W. L. 1994. Molecularmarker-facilitated investigation of host-plant response to Exserohilum turcicum in maize (Zea mays L): Components of resistance. Theor. Appl. Genet. 88: 305-313.

Gai, J. Y., and Wang, J. K. 1998. Identification and estimation of a QTL model and its effects. Theor. Appl. Genet. 97:1162-1168.

Gevers, H. O., Lake, J. K., and Hohls, T. 1994. Diallel cross analysis of resistance to gray leaf spot in maize. Plant Dis. 78:379-383.

Goodman, M. M. 2004. Developing temperate inbreds using tropical maize germplasms: Rationale, results, conclusions. Maydica 49:209-219.

Gordon, S. G., Bartsch, M., Matthies, I., Gevers, H. O., Lipps, P. E., and Pratt, R. C. 2004. Linkage of molecular markers to Cercospora zeae-maydis resistance in maize. Crop Sci. 44:628-636.

Gordon, S. G., Lipps, P. E., and Pratt, R. C. 2006. Heritability and components of resistance to Cercospora zeae-maydis derived from maize inbred VO613Y. Phytopathology 96:593-598.

Huff, C. A., Ayers, J. E., and Hill, R. R., Jr. 1988. Inheritance of resistance in corn (Zea mays) to gray leaf spot. Phytopathology 78:790-794.

Jayaramachandran, M., Kumaravadivel, N., Eapen, S., and Kandasamy, G. 2010. Gene action for yield attributing characters in segregating generation (M2) of sorghum (Sorghum bicolor L.). Electron J. Plant Breed. 1:802-805.

Katwal, T. B., Wangchuk, D., Dorji, L., Wangdi, N., and Choney, R. 2013. Evaluation of gray leaf spot tolerant genotypes from CIMMYT in the highland maize production eco-systems of Bhutan. J. Life Sci. 7:443-452.

Kosambi, D. D. 1943. The estimation of distances from recombination values. Ann. Eugen. 12:172-175.

Latterell, F. M., and Rossi, A. E. 1983. Gray leaf spot of corn: A disease on the move. Plant Dis. 67:842-847.

Lefebvre, V., and Palloix, A. 1996. Both epistatic and additive effects of QTLs are involved in polygenic induced resistance to disease: A case study, the interaction pepper-Phytophthora capsici Leonian. Theor. Appl. Genet. 93: 503-511.

Lehmensiek, A., Esterhuizen, A. M., Van Staden, D., Nelson, S. W., and Retief, A. E. 2001. Genetic mapping of gray leaf spot (GLS) resistance genes in maize. Theor. Appl. Genet. 103:797-803.

Liu, K. J., and Xu, X. D. 2013. First report of gray leaf spot of maize caused by Cercospora zeina in China. Plant Dis. 97:1656.

McCouch, S. R., Teytelman, L., Xu, Y., Lobos, K. B., Clare, K., Walton, M., Fu, B., Maghirang, R., Li, Z., Xing, Y., Zhang, Q., Kono, I., Yano, M., Fjellstrom, R., DeClerck, G., Schneider, D., Cartinhour, S., Ware, D., and Stein, L. 2002 Development and mapping of 2240 new SSR markers for rice (Oryza sativa L.). DNA Res. 9:199-207.

McGee, D. C. 1988. Maize Diseases: A Reference Source for Seed Technologists American Phytopathological Society, St. Paul, MN.

McLachlan, G. J., and Basford, K. E. 1988. Mixture Models: Inference and Applications to Clustering. Marcel Dekker, Inc., New York.

Menkir, A., and Ayodele, M. 2005. Genetic analysis of resistance to gray leaf spot of mid altitude maize inbred lines. Crop Sci. 45:163-170.

Messmer, R., Fracheboud, Y., Banziger, M., Vargas, M., Stamp, P., and Ribaut, J. M. 2009. Drought stress and tropical maize: QTL-by-environment interactions and stability of QTLs across environments for yield components and secondary traits. Theor. Appl. Genet. 119:913-930. 
Murray, D., Payne, R., Welham, S., and Zhang, Z. Z. 2013. Breeding View: A Visual Tool for Running Analytical Pipelines. VSN International Ltd., Hemel Hempstead, UK.

Nzuve, F., Githiri, S., Mukunya, D. M., and Gethi, J. 2013. Combining abilities of maize inbred lines for grey leaf spot (GLS), grain yield and selected agronomic traits in Kenya. J. Plant Breed. Crop Sci. 5:41-47.

Pozar, G., Butruille, D., Silva, H. D., McCuddin, Z. P., and Penna, J. C. V. 2009. Mapping and validation of quantitative trait loci for resistance to Cercospora zeae-maydis infection in tropical maize (Zea mays L.). Theor. Appl. Genet. 118:553-564.

Saghai Maroof, M. A., Van Scoyoc, S. W., Yu, Y. G., and Stromberg, E. L. 1993. Gray leaf spot disease of maize; rating methodology and inbred line evaluation. Plant Dis. 77:583-587.

Saghai Maroof, M. A., Yue, Y. G., Xiang, Z. X., Stromberg, E. L., and Rufener, G. K. 1996. Identification of quantitative trait loci controlling resistance to gray leaf spot disease in maize. Theor. Appl. Genet. 93:539-546.

Salvi, S., Sponza, G., Morgante, M., Tomes, D., Niu, X., Fengler, K. A., Meeley, R., Ananiev, E. V., Svitashev, S., Bruggemann, E., Li, B., Hainey, C. F., Radovic, S., Zaina, G., Rafalski, J. A., Tingey, S. V., Miao, G. H., Phillips, R. L., and Tuberosa, R. 2007. Conserved noncoding genomic sequences associated with a flowering-time quantitative trait locus in maize. Proc. Natl. Acad. Sci. USA 104:11376-11381.

Samak, N. R. A., Hittalmani, S., Shashidhar, N., and Biradar, H. 2011. Exploratory studies on genetic variability and genetic control for protein and micronutrient content in $\mathrm{F}_{4}$ and $\mathrm{F}_{5}$ generation of rice (Oryza sativa L.). Asian J. Plant Sci. 10:376-379.

SAS Institute Inc. 2004. SAS/STAT User's Guide. SAS Institute Inc., Cary, NC.

Shi, L. Y., Li, X. H., Hao, F., Xie, C. X., Ji, H. L., Lv, X. L., Zhang, S. H., and Pan, G. T. 2007. Comparative QTL mapping of resistance to gray leaf spot in maize based on bioinformatics. Agric. Sci. China 6:1411-1419.

Shi, L. Y., Lv, X. L., Weng, J. F., Zhu, H. Y., Liu, C. L., Hao, Z. F., Zhou, Y., Zhang, D. G., Li, M. S., Ci, X. K., and Li, X. H. 2014. Genetic characterization and linkage disequilibrium mapping of resistance to gray leaf spot in maize (Zea mays L.). Crop J. 2:132-143.

Sriwatanapongse, S., Jinahyon, S., and Vasal, S. K. 1993. Suwan-1: Maize from Thailand to the World. CIMMYT, D.F., Mexico.

Tehon, L. R., and Daniels, E. 1925. Notes on the parasitic fungi of Illinois. Mycologia 17:240-249.
Wang, C., Ulloa, M., Mullens, T. R., Yu, J., and Roberts, P. A. 2012. QTL analysis for transgressive resistance to root-knot nematode in interspecific cotton (Gossypium spp.) progeny derived from susceptible parents. PLoS One 7: e34874.

Wang, J. K., and Gai, J. Y. 2001. Mixed inheritance model for resistance to agromyzid beanfly (Melanagromyza sojae Zehntner) in soybean. Euphytica 122:9-18

Wang, J. K., Li, H. H., Zhang, L. Y., Li, C. H., and Meng, L. 2011. QTL IciMapping v3.1. Institute of Crop Sciences, CAAS, Beijing, China and Crop Research Informatics Laboratory, CIMMYT, Apdo, D.F., Mexico.

Ward, J. M. J., Stromberg, E. L., Nowell, D. C., and Nutter, F. W., Jr. 1999. Gray leaf spot: A disease of global importance in maize production. Plant Dis. 83: 884-895.

Wu, J. Z., Liu, S. J., Sha, B. C., Lu, C. H., Mao, X. Q., and He, Y. Q. 2009. Establishment of assessment standard for gray leaf spot of corn using $\mathrm{F}_{2}$ populations. Acta Phytophylac. Sin. 36:479-480. (In Chinese with English abstract in Chinese)

Xu, L., Zhang, Y., Shao, S. Q., Chen, W., Tan, J., Zhu, M., Zhong, T., Fan, X. M., and $\mathrm{Xu}, \mathrm{M}$. L. 2014. High-resolution mapping and characterization of $q R g l s 2$, major quantitative trait locus involved in maize resistance to gray leaf spot. BMC Plant Biol. 14:230.

Young, N. D., Danesh, D., Menancio-Hautea, D., and Kumar, L. 1993. Mapping oligogenic resistance to powdery mildew in mung bean with RFLPs. Theor. Appl. Genet. 87:243-249.

Zhang, Y., Xu, L., Fan, X., Tan, J., Chen, W., and Xu, M. 2012. QTL mapping of resistance to gray leaf spot in maize. Theor. Appl. Genet. 125:1797-1808

Zhang, Y. M., Gai, J. Y., and Yang, Y. H. 2003. The EIM algorithm in the joint segregation analysis of quantitative traits. Genet. Res. 81:157-163.

Zhang, Y.-M., and Xu, S. 2004. Mapping quantitative trait loci in $\mathrm{F}_{2}$ incorporating phenotypes of $\mathrm{F}_{3}$ progeny. Genetics 166:1981-1993.

Zwonitzer, J. C., Coles, N. D., Krakowsky, M. D., Arellano, C., Holland, J. B., McMullen, M. D., Pratt, R. C., and Balint-Kurti, P. J. 2010. Mapping resistance quantitative trait Loci for three foliar diseases in a maize recombinant inbred line population-evidence for multiple disease resistance? Phytopathology 100:72-79. 